\title{
Uso de clinoptilolita como un vehículo de fertilizantes nitrogenados en un suelo de la región Pampeana de Argentina
}

\author{
Guillermo Millán ${ }^{1}$, Florencia Agosto², Mabel Vázquez, Lia Botto², \\ Luciano Lombardi ${ }^{1}$ y Luciano Juan ${ }^{1}$ \\ 'Cátedra de Edafología, Facultad de Ciencias Agrarias y Forestales, Universidad Nacional de La Plata. \\ Calle 60 y 119 (1900). La Plata, Argentina. \\ ${ }^{2}$ Cátedra de Química Inorgánica, Facultad de Ciencias Exactas, Universidad Nacional de La Plata. \\ Calle 47 y 115 (1900). La Plata, Argentina.
}

\begin{abstract}
G. Millán, F. Agosto, M. Vázquez, L. Botto, L. Lombardi, and L. Juan. 2008. Use of clinoptilolite as a carrier for nitrogen fertilizers in soils of the Pampean regions of Argentina. Cien. Inv. Agr. 35(3):293-302. The properties of clinoptilolite related to cationic exchange and ionic selectivity have motivated us to study its application for agronomic purposes. The high potential of the clinoptilolite has been demonstrated as a vehicle for nitrogen fertilizers, with the aims of decreasing negative impacts on the environment and increasing fertilizer efficiency. The objective of this study was to evaluate the effects of an exchanged form, clinoptilolite$\mathrm{NH}_{4}$, and an occluded form, clinoptilolite-urea, of nitrogen fertilizer relative to commercial urea on dry matter and the nitrogen uptake efficiency of Lolium multiflorum under controlled conditions. Lolium multiflorum, sowed in a sandy loam soil, was fertilized with $0,60,120$, and $180 \mathrm{~kg} \cdot \mathrm{ha}^{-1}$ of nitrogen. Yield increased considerably and nitrogen uptake efficiency was enhanced when clinoptilolite- $\mathrm{NH}_{4}$ was used, possibly due to its ability to retain and slowly liberate $\mathrm{NH}_{4}^{+}$ions. This effect produces time differences in nitrogen disponibility in relation to urea, making the nitrogen available for a longer time for the plant. The clinoptilolite-urea treatments reduced yields significantly in comparison with urea applications only at the lowest dose. In addition, the nitrogen uptake efficiency was always lower than the other treatments. This behavior is related to the fact that nitrogen liberation dynamics of the occluded form are much slower than for the ionic one.
\end{abstract}

Key words: Cation desorption, molecular occlusion, $\mathrm{N}$ uptake efficiency.

\section{Introducción}

La dinámica del nitrógeno en sistemas sueloaire-agua ha sido objeto de gran interés en la literatura científica, debido al relevante papel que desempeña en la producción agrícola y por la importancia de un uso racional del nitrógeno. El aumento de la eficiencia del uso del nitrógeno, debida al uso de tecnologías de suministro gradual de nitrógeno, tiene repercusión sobre la producción vegetal y es un factor fundamental para reducir la contaminación del ambiente

Recibido 28 diciembre 2007. Aceptado 08 mayo 2008.

'Dirigir correspondencia a G. Millán: gmillan@ agro.unlp.edu.ar
(Bolado Rodríguez et al., 2003). En Argentina se ha demostrado la contaminación de aguas subterráneas y superficiales como consecuencia de la fertilización nitrogenada (Costa, 1997; Andriulo et al., 2000). Por lo tanto, el desarrollo de tecnologías de fertilización de liberación lenta de nitrógeno podría disminuir la contaminación y mejorar los rendimientos de los cultivos.

La clinoptilolita es la zeolita natural más abundante en la naturaleza. Posee una alta capacidad de intercambio catiónico y una gran afinidad por los iones $\mathrm{NH}_{4}^{+}$(Inglesakis, 2004). Aunque existe cierta controversia, se atribuye a las zeolitas en general, y particularmente a las 
clinoptilolitas, la capacidad de retener y liberar lentamente los iones $\mathrm{NH}_{4}^{+}$que se incorporan en la red de canales que forman su estructura cristalina (Lewis et al., 1984; Ferguson and Pepper, 1987; Mack Kown and Tucker., 1985; Allen et al., 1996; Kithome et al., 1998). En trabajos previos se demostró una alta potencialidad de estos minerales como vehículo de fertilizantes nitrogenados, con la finalidad de disminuir problemas ambientales y aumentar la eficiencia de los fertilizantes. Asimismo, existe la posibilidad de utilizar la clinoptilolita como un intercambiador molecular. La dinámica de liberación de moléculas nitrogenadas ocluídas en la clinoptilolita difiere de la de iones. Las moléculas nitrogenadas quedan retenidas por atracción electroestática y se producen en ella modificaciones en sus ángulos moleculares $\mathrm{y}$ en los enlaces simples y dobles (Park y Komarneni, 1998).

La dinámica suelo-clinoptilolita-nitrógeno es variable, dependiendo de las características fisicoquímicas de los suelos, de la dosis de clinoptilolita y nitrógeno aplicada, del manejo del cultivo y de la época del año en la cual se realizan los ensayos (Kolyagin y Karasev, 1999; Postnikov et al., 1996; Ando et al., 1996; Babaririck y Pirela, 1984; Lewis et al., 1984). Este hecho sugiere la necesidad de hacer evaluaciones en los suelos donde se emplearán.

En trabajos previos la utilización de clinoptilolita ha permitido aumentar la eficienciade utilización de fertilizantes nitrogenados. Asimismo ha permitido obtener altos rendimientos con menores dosis de nitrógeno (Soca et al., 2004; Loboda, 1999). Por lo tanto, es posible que el aporte de nitrógeno con soporte de clinoptilolita a un suelo, permita aumentar la eficiencia de uso del fertilizante nitrogenado en especies forrajera, en relación con una fertilización con urea.

El objetivo de este trabajo fue evaluar la producción de materia seca y la recuperación de nitrógeno del fertilizante en Lolium multiflorum, cultivado bajo cubierta en un suelo franco arenoso, fertilizado con clinoptilolita$\mathrm{NH}_{4}$ y clinoptilolita-urea, en comparación con una fertilización nitrogenada tradicional con urea granulada.

\section{Materiales y métodos}

\section{Obtención y características de la clinoptilolitas}

Las clinoptilolitas utilizadas se obtuvieron en un yacimiento de zeolita de La Rioja, Argentina. Correspondieron a clinoptilolitas sódicas, de origen vulcanoclástico, con alta capacidad de intercambio catiónico (Chelishehev et al., 1988) (Cuadro 1).

Se molieron y tamizaron a un diámetro de partículas entre 1 y $2 \mathrm{~mm}$. Para obtener la forma monoiónica amónica, se utilizaron un matraz de $500 \mathrm{ml}$ donde se trató $50 \mathrm{~g}$ de clinoptilolita con $500 \mathrm{~mL}$ de $\mathrm{NH}_{4} \mathrm{Cl}$ 0,5 $\mathrm{N}$ en relación 1:10 (clinoptilolita:solución nitrogenada de intercambio). Este proceso se prolongó por $96 \mathrm{~h}$ a $20^{\circ} \mathrm{C}$ $\left( \pm 2^{\circ} \mathrm{C}\right)$. Se evaluó el $\mathrm{NH}_{4}^{+}$retenido por destilación Kjedhal. El $\mathrm{NH}_{4}^{+}$que ocuparon los sitios de intercambio de la clinoptilolita se calculó por la diferencia entre la concentración inicial y final de $\mathrm{NH}_{4}^{+}$en el sobrenadante (Inglezakis et al., 2004).

Las clinoptilolitas combinadas con urea se obtuvieron tratando $50 \mathrm{~g}$ de clinoptilolita con $500 \mathrm{~mL}$ de $\mathrm{HCl} 0,5 \mathrm{~N}$ en un matraz de 500 $\mathrm{mL}$. Este proceso se prolongó por $96 \mathrm{~h}$ a $20^{\circ} \mathrm{C}$ $\left( \pm 2^{\circ} \mathrm{C}\right)$ de modo de obtener una clinoptilolita ácida (clinoptilolita-H) libre de cationes. La solución de intercambio se renovó cada $48 \mathrm{~h}$ (Inglezakis et al., 2004).

En el segundo paso se obtuvo la clinoptilolitaurea por intercambio en lechos de contacto de urea fundida a $200^{\circ} \mathrm{C}$ con clinoptilolita-H. Se calculó la urea incorporada en la clinoptilolita por diferencia de peso.

\section{Suelo, muestreo y procesamiento}

El suelo utilizado se obtuvo de los primeros 20 $\mathrm{cm}$ del horizonte superficial de un Hapludol Típico (Lincoln, Buenos Aires, Argentina), perteneciente a la Pradera Pampeana de la subregión Pampa Arenosa. El suelo se secó al aire, se desagregó, se tamizó (malla de $2 \mathrm{~mm}$ ) y se caracterizó física y químicamente según SAGPyA, (Secretaría de Agricultura, Ganadería y Pesca de la Nación y la Asociación Argentina de la Ciencia del Suelo) (Cuadro 2). 
Cuadro 1. Caracterización fisicoquímica del suelo y la clinoptilolita.

Table 1. Soil and clinoptilolite physicochemical analysis.

\begin{tabular}{|c|c|}
\hline Parámetros & Valor \\
\hline \multicolumn{2}{|l|}{ Suelo } \\
\hline $\mathrm{pH}^{1}$ & 5,60 \\
\hline Conductividad eléctrica, $\mathrm{dSm} \cdot \mathrm{m}^{-1}$ & 0,80 \\
\hline $\begin{array}{l}\text { Capacidad intercambio catiónico, } \\
(\mathrm{CIC})^{2}, \mathrm{cmolc} \cdot \mathrm{kg}^{-1}\end{array}$ & 15,20 \\
\hline $\mathrm{Ca}^{+2}$ intercambiable, cmolc $\cdot \mathrm{kg}^{-1}$ & 10,20 \\
\hline $\mathrm{Mg}^{+2}$ intercambiable, cmolc $\cdot \mathrm{kg}^{-1}$ & 1,50 \\
\hline $\mathrm{Na}^{+}$intercambiable, cmolc $\cdot \mathrm{kg}^{-1}$ & 0,50 \\
\hline $\mathrm{K}^{+}$intercambiable, cmolc $\cdot \mathrm{kg}^{-1}$ & 1,80 \\
\hline MO (Walkley-Black), $\mathrm{mg} \cdot \mathrm{kg}^{-1}$ & 34,00 \\
\hline $\mathrm{N}, \%$ & 0,19 \\
\hline Relación C/N & 10,50 \\
\hline $\mathrm{P}\left(\right.$ Bray-Kurtz No $\left.{ }^{\circ} 1\right), \mathrm{mg} \cdot \mathrm{kg}^{-1}$ & 19,00 \\
\hline \multicolumn{2}{|l|}{ Clase textural: Franco arenoso } \\
\hline Arena, $\%$ & 53,00 \\
\hline Limo, $\%$ & 31,00 \\
\hline Arcilla, & 16,00 \\
\hline \multicolumn{2}{|l|}{ Retención hídrica: } \\
\hline $3 \mathrm{MPa}, \%$ & 25,00 \\
\hline $147 \mathrm{MPa}, \%$ & 9,00 \\
\hline \multicolumn{2}{|l|}{ Clinoptilolita } \\
\hline $\mathrm{SiO}_{2}, \%$ & 62,70 \\
\hline $\mathrm{Al}_{2} \mathrm{O}_{3}, \%$ & 12,50 \\
\hline $\mathrm{Fe}_{2}^{2} \mathrm{O}_{3}, \%$ & 0,60 \\
\hline $\mathrm{CaO}, \%$ & 0,40 \\
\hline $\mathrm{MgO}, \%$ & 0,60 \\
\hline $\mathrm{Na}_{2} \mathrm{O}, \%$ & 6,40 \\
\hline $\mathrm{MnO}, \%$ & 0,01 \\
\hline $\mathrm{K}_{2} \mathrm{O}, \%$ & 1,20 \\
\hline $\mathrm{P}_{2} \mathrm{O}_{5}, \%$ & 0,03 \\
\hline $\mathrm{CiC}^{2}, \mathrm{cmolc}^{2} \mathrm{~kg}^{-1}$ & 175,00 \\
\hline
\end{tabular}

${ }^{1} \mathrm{pH}$ determinado en extracto de suelo en relación 1:2,5, suelo:agua. ${ }^{2}$ Capacidad de intercambio catiónico (CIC) evaluada a pH 6.

${ }^{\mathrm{I}} \mathrm{pH}$ was determined in soil 1:2.5 (soil:water) soil extract.

${ }^{2}$ Cationic exchange Capacity (CEC) evaluated for $\mathrm{pH} 6$.

\section{Ensayo de invernáculo}

Se realizó un ensayo en invernáculo donde los tratamientos fueron el tipo de fertilizante (clinoptilolita- $\mathrm{NH}_{4}$, clinoptilolita-urea y urea) y las dosis de nitrógeno $(0,60,120$ y $180 \mathrm{~kg} \cdot \mathrm{ha}$ ${ }^{1}$ de nitrógeno). Las unidades experimentales fueron macetas de $0,4 \mathrm{~L}$ con $300 \mathrm{~g}$ de suelo. En función de la concentración de nitrógeno de la clinoptilolita modificada, las dosis de nitrógeno empleadas derivaron en relaciones $(\mathrm{p} / \mathrm{p})$ suelo: clinoptilolita de 1:0; 1:0,0003; 1:0,0006 y 1:0,0012 para las dosis de $0,60,120$ y 180 $\mathrm{kg} \cdot \mathrm{ha}^{-1}$ de nitrógeno, respectivamente.
Se fertilizó y se regó de modo de mantener el suelo a $90 \%$ de capacidad de campo (3 MPa), mediante riegos realizados con agua destilada cada $48 \mathrm{~h}$. Se sembraron 130 semilla por maceta de L. multiflorum cv. Grandesa tetraploide. Pos-emergencia, se ralearon para dejar 100 planta.maceta ${ }^{-1}$. A partir de la emergencia, se realizaron cuatro cortes, al ras de la maceta, cada 28 d. En cada ocasión se determinó la materia seca (peso constante a $60^{\circ} \mathrm{C}$ ) y nitrógeno (digestión húmeda en medio sulfúrico con $\mathrm{H}_{2} \mathrm{O}_{2}$ y valoración Kjeldahl) (Malavolta et al., 1989).

Se calculó la eficiencia de recuperación del nitrógeno del fertilizante (ERNF), utilizando la fórmula:

$\mathrm{ERNF}=[(\mathrm{N}$ absorbido tratamiento $-\mathrm{N}$ absorbido testigo) $\mathrm{x}$ (dosis de $\mathrm{N}$ aplicado) ${ }^{-1}$ x 100.

\section{Diseño y análisis estadísticos}

El diseño estadístico fue en bloques completos al azar con arreglo factorial $3 \times 4$, con tres repeticiones de cada tratamiento. Los factores fueron tres tipos de fertilizante (clinoptilolita$\mathrm{NH}_{4}$, clinoptilolita-urea y urea) y los subfactores cuatro dosis de nitrógeno equivalentes a $0,60,120$ y $180 \mathrm{~kg} \cdot \mathrm{ha}^{-1}$. Se realizó un análisis de varianza y los promedios se separaron de acuerdo con la prueba de las diferencias mínimas significativas (LSD) (Mendenhall et al., 1986).

\section{Resultados y discusión}

\section{Producción de materia seca}

En cuatro cortes realizados, la producción de materia seca total aumentó significativamente para todas las dosis de nitrógeno empleadas cuando se utilizó clinoptilolita- $\mathrm{NH}_{4}$, en comparación con la utilización de urea granulada (Figura 1E). Independientemente de la forma de utilización de la clinoptilolita, este aumento de rendimiento coincidió con lo expuesto en trabajos previamente realizados (Pirela et al., 1984; Kolyagin y Karasev, 1999; Soca et al., 2004).

En relación con urea que obtuvo un $13,25 \%$ de incremento en rendimientos, al utilizar 
Cuadro 2. Nitrógeno total $(\mathrm{N})$ en la parte aérea de Lolium multiflorum.

Table 2. Total nitrogen $(N)$ in the aerial plant part of Lolium multiflorum.

\begin{tabular}{|c|c|c|c|c|c|}
\hline \multirow[t]{2}{*}{ Tipo de fertilizante } & \multirow{2}{*}{$\begin{array}{c}\text { Dosis de N } \\
\mathrm{kg} \cdot \mathrm{ha}^{-1}\end{array}$} & \multicolumn{4}{|c|}{$\mathrm{N}$ total en la parte aérea, mg } \\
\hline & & Corte 1 & Corte 2 & Corte 3 & Total \\
\hline Clinoptilolite- $\mathrm{NH}_{4}$ & 0 & $5,0 \mathrm{a}^{1}$ & $2,2 \mathrm{a}^{1}$ & $1,0 \mathrm{a}^{1}$ & $8,1 \mathrm{a}^{1}$ \\
\hline Clinoptilolite- $\mathrm{NH}_{4}$ & 60 & $11,9 \mathrm{c}$ & $5,5 \mathrm{c}$ & $1,8 \mathrm{c}$ & $20,2 \mathrm{c}$ \\
\hline Clinoptilolite- $\mathrm{NH}_{4}^{4}$ & 120 & 16,5 ef & $12,0 \mathrm{f}$ & $3,5 \mathrm{e}$ & $31,9 \mathrm{f}$ \\
\hline Clinoptilolite- $\mathrm{NH}_{4}^{4}$ & 180 & $18,3 \mathrm{f}$ & $17,1 \mathrm{~g}$ & $5,1 \mathrm{f}$ & $40,5 \mathrm{~h}$ \\
\hline Clinoptilolite-urea & 0 & $5,0 \mathrm{a}$ & $2,2 \mathrm{a}$ & $1,0 \mathrm{a}$ & $8,1 \mathrm{a}$ \\
\hline Clinoptilolite-urea & 60 & $9,3 \mathrm{~b}$ & $4,4 \mathrm{~b}$ & $1,2 \mathrm{ab}$ & $14,9 \mathrm{~b}$ \\
\hline Clinoptilolite-urea & 120 & $12,9 \mathrm{c}$ & $9,0 \mathrm{~d}$ & $2,1 \mathrm{~cd}$ & $24,0 \mathrm{~d}$ \\
\hline Clinoptilolite-urea & 180 & $13,4 \mathrm{~cd}$ & $10,6 \mathrm{e}$ & $3,5 \mathrm{e}$ & $27,5 \mathrm{e}$ \\
\hline Urea & 0 & $5,0 \mathrm{a}$ & $2,2 \mathrm{a}$ & $1,0 \mathrm{a}$ & $8,1 \mathrm{a}$ \\
\hline Urea & 60 & $15,0 \mathrm{de}$ & $4,1 \mathrm{~b}$ & $1,4 \mathrm{~b}$ & $20,5 \mathrm{c}$ \\
\hline Urea & 120 & $18,5 \mathrm{f}$ & $7,6 \mathrm{c}$ & $1,9 \mathrm{c}$ & $28,0 \mathrm{e}$ \\
\hline Urea & 180 & $22,7 \mathrm{~g}$ & $10,0 \mathrm{e}$ & $2,5 \mathrm{~d}$ & $35,3 \mathrm{~g}$ \\
\hline
\end{tabular}

clinoptilolita- $\mathrm{NH}_{4}$ se obtuvo 13,25 y $26 \%$ de aumento de rendimiento en la medida que aumentó la dosis de nitrógeno, respectivamente (Figura 1E). Al analizar la producción total de materia seca, la interacción entre tipo de fertilizante nitrogenado y la dosis fue significativa ( $<<0,001)$ (Cuadro 3). Asimismo, se observó que la utilización de este tipo de fertilizante permitió obtener rendimientos estadísticamente similares a urea aplicada en dosis mayores. Por ejemplo, cuando se aplicaron $120 \mathrm{~kg} \cdot \mathrm{ha}^{-1}$ utilizando clinoptilolita$\mathrm{NH}_{4}$ se obtuvieron rendimientos equivalentes a los obtenidos con la utilización de urea en la dosis de $180 \mathrm{~kg} \cdot \mathrm{ha}^{-1}$.

Al analizar la producción por cada corte de L. multiflorum, se observó que para la dosis de $60 \mathrm{~kg} \cdot \mathrm{ha}^{-1}$ los tratamientos fertilizados con clinoptilolita- $\mathrm{NH}_{4}$ y urea no registraron diferencias significativas. A dosis mayores existió un comportamiento diferencial según el tipo de fertilizante utilizado y el corte analizado. Para el primer corte los tratamientos fertilizados con urea exhibieron una mayor producción de materia seca para las dosis de 120 y $180 \mathrm{~kg} \cdot \mathrm{ha}^{-1}$ (Figura 1A). En el segundo corte se determinó una mayor producción de los tratamientos fertilizados con clinoptilolita- $\mathrm{NH}_{4}$ sólo en la dosis de $180 \mathrm{~kg} \cdot \mathrm{ha}^{-1}$ (Figura 1B). En el tercer y cuarto corte el aumento de la producción de materia seca se registró para las dosis de 120 y $180 \mathrm{~kg} \cdot \mathrm{ha}^{-1}$ cuando se utilizó clinoptilolita$\mathrm{NH}_{4}$ (Figura 1C, D). Las interacciones entre el tipo de fertilizante nitrogenado y la dosis empleada fueron significativas $(\mathrm{p}<0,004)$ para el segundo, tercer y cuarto corte (Cuadro 3).

En las condiciones en que se realizó el ensayo, con un suelo mantenido a $80 \%$ de capacidad de campo y sin percolación, las perdidas por volatilización de $\mathrm{NH}_{3}$ serían bajas y no se producirían perdidas por lixiviación de $\mathrm{NO}_{3}$. Por lo cual, se atribuye el aumento de rendimiento de materia seca a la capacidad de retención de iones $\mathrm{NH}_{4}^{+}$que poseen las clinoptilolitas y a la lenta liberación, permitiendo la existencia de iones $\mathrm{NH}_{4}^{+}$en la solución del suelo por un periodo mayor de tiempo.

Watson (1986) demostró que estas plantas pueden absorber preferencialmente amonio y que su crecimiento vegetativo se ve favorecido en un medio con una baja relación $\mathrm{NO}_{3}^{-}: \mathrm{NH}_{4}^{+}$ (Griffith y Streeter, 1994). Esto indicaría que el $\mathrm{NH}_{4}^{+}$que ocupa los canales internos de la clinoptilolita sería liberado lentamente, permitiendo una absorción progresiva por el cultivo que redunda en una mayor producción de materia seca a lo largo del cultivo. Este efecto sobre los rendimientos se observa con mayor magnitud en suelos con baja capacidad de intercambio catiónico (CIC) y textura gruesa, como ocurrió en el suelo utilizado en el presente ensayo (Ferguson y Pepper, 1987).

La producción de materia seca total de $L$. multiflorum obtenida en los tratamientos 

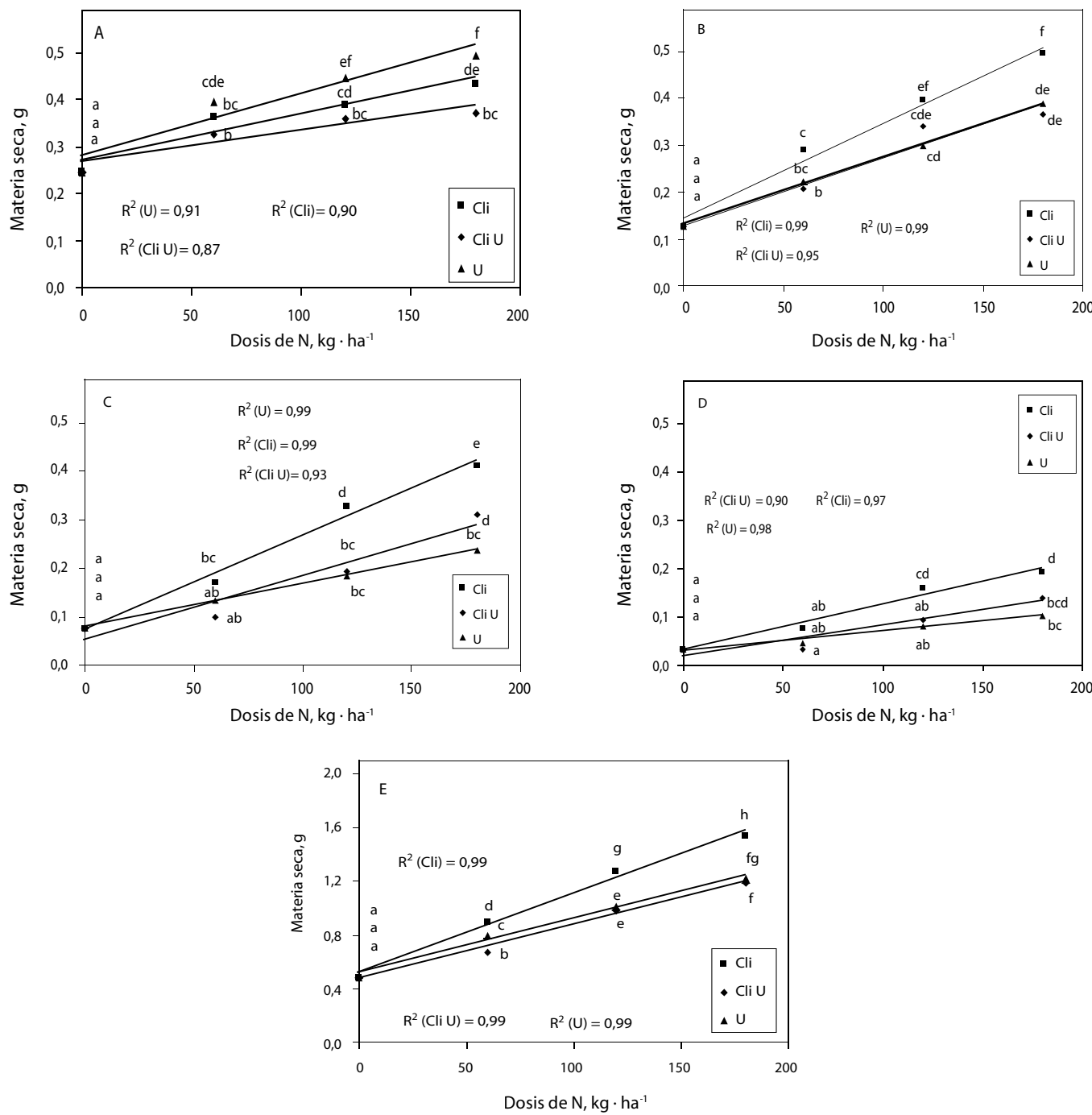

Figura 1. Efecto de tres tipos de fertilizantes nitrogenados sobre los rendimientos de materia seca de Lolium multiflorum cv. Grandeza. A. Primer corte. B. Segundo corte. C. Tercer corte. D. Cuarto corte. E. Total acumulado en los cuatro cortes. Promedios en cada dosis de nitrógeno $(\mathrm{N})$ seguidos por iguales letras no son estadísticamente diferentes entre sí según la prueba de comparación múltiple de medias LSD ( $<<0,05)$. Cli = clinoptilolita-NH4 y Cli U = clinoptilolita-Urea. U= urea. Figure 1. Dry matter of Lolium multiflorum $c v$. Grandeza obtained in relation to three types of nitrogen fertilizer in the first cut (A), second cut (B), third cut $(C)$ and fourth cut $(D)$, and the total accumulated yields $(E)$. Means for each nitrogen $(N)$ doses followed by the same letters are not statistically different from each other according to the least significant differences $(L S D)$ test $(p=0.05)$. Cli $=$ Clinoptilolite-NH4, Cli $U=$ Clinoptilolite-urea, and $U=$ urea .

fertilizados con clinoptilolita-urea, registró valores similares de producción de materia seca para las dosis de 120 y $180 \mathrm{~kg} \cdot \mathrm{ha}^{-1}$. Sin embargo, para la dosis de $60 \mathrm{~kg} \cdot \mathrm{ha}^{-1}$ la producción de materia seca fue menor a la del tratamiento fertilizado con urea (Figura 1E).
Al analizar la producción por cortes de $L$. multiflorum, la producción de materia seca fue inferior para todas las dosis de nitrógeno en el primer corte, en relación a la urea (Figura 1A). En el segundo corte no se observaron diferencias estadísticamente significativas para las distintas dosis analizadas (Figura 1B). En 
el tercer corte sólo se observó diferencia para la dosis de $180 \mathrm{~kg} \cdot \mathrm{ha}^{-1}$, a favor de la utilización de clinoptilolita-urea (Figura 1C). En el cuarto corte no se registraron diferencias entre los distintos tratamientos (Figura 1D). Esto indicaría que la urea ocluida en la clinoptilolita, estaría fuertemente retenida, presentando una dinámica de liberación más lenta, si se la compara con la retención de iones (Park y Komarnemi, 1998).

\section{Nitrógeno total de materia seca}

El contenido de nitrógeno total (NT) en la parte aérea de L. multiflorum presentó una marcada diferencia en relación a las dos formas de clinoptilolitas analizadas. Independientemente de la fecha de corte de L. multiflorum, la interacción entre el efecto del tipo de fertilizante nitrogenado y la dosis aplicada fue significativa ( $<<0,001)$ (Cuadro 3). Cuando se utilizó clinoptilolita- $\mathrm{NH}_{4}$ el contenido de NT en la parte aérea coincidió con la producción de materia seca, analizada en el punto anterior. Para la dosis más baja $\left(60 \mathrm{~kg} \cdot \mathrm{ha}^{-1}\right)$ no se observan diferencias en los contenidos de NT. Sin embargo, para las dosis mayores $\left(120 \mathrm{y} 180 \mathrm{~kg} \cdot \mathrm{ha}^{-1}\right)$ se observó que la utilización de clinoptilolita- $\mathrm{NH}_{4}$ permitió incrementar el NT en relación a la urea. Por el contrario, el contenido de NT de los tratamientos fertilizados con clinoptilolita-urea fue inferior a la de los tratamientos fertilizados directamente con urea.

$\mathrm{Al}$ analizar NT por cortes de L. multiflorum se observó que en el primer corte, fue la urea el tipo de fertilizante que permitió obtener una mayor disponibilidad de nitrógeno. Esta diferencia se observó para todas las dosis analizadas en relación a la clinoptilolita-urea, y para las dosis de 60 y $180 \mathrm{~kg} \cdot \mathrm{ha}^{-1}$ si la comparación

Cuadro 3. Analisis de varianza para rendimiento en material y eficiencia de recuperación del nitrógeno del fertilizante (ERNF) obtenido con Lolium multiflorum en cuatro cortes y respecto del total.

Table 3. Analysis of variance for dry matter yield, total nitrogen content, and efficiency of nitrogen retrieval from nitrogen fertilizer (ENRF) obtained for Lolium multiflorum in four cuts and in total.

\begin{tabular}{|c|c|c|c|c|c|c|}
\hline \multirow[b]{2}{*}{ Fuente de variación } & \multicolumn{2}{|c|}{ Rendimiento en material seca } & \multicolumn{2}{|c|}{ Nitrógeno total } & \multicolumn{2}{|c|}{ ERNF $^{1}$} \\
\hline & $\mathrm{F}$ & $\mathrm{p}$ & $\mathrm{F}$ & $\mathrm{p}$ & $\mathrm{F}$ & $\mathrm{p}$ \\
\hline \multicolumn{7}{|l|}{ Corte 1} \\
\hline Tipo (T) & 15,64 & $<0,001$ & 95,54 & $<0,001$ & 86,51 & $<0,001$ \\
\hline Dosis (D) & 63,37 & $<0,001$ & 356,60 & $<0,001$ & 41,65 & $<0,001$ \\
\hline $\mathrm{D} \times \mathrm{T}$ & 2,19 & 0,08 & 13,44 & $<0,001$ & 5,44 & 0,005 \\
\hline \multicolumn{7}{|l|}{ Corte 2} \\
\hline Tipo $(\mathrm{T})$ & 25,53 & $<0,001$ & 117,83 & $<0,001$ & 93,65 & $<0,001$ \\
\hline Dosis (D) & 203,25 & $<0,001$ & 549,47 & $<0,001$ & 13,04 & $<0,001$ \\
\hline $\mathrm{D} \times \mathrm{T}$ & 4,25 & 0,004 & 23,87 & $<0,001$ & 0,70 & 0,603 \\
\hline \multicolumn{7}{|l|}{ Corte 3} \\
\hline Tipo (T) & 111,88 & $<0,001$ & 135,12 & $<0,001$ & 95,03 & $<0,001$ \\
\hline Dosis (D) & 417,58 & $<0,001$ & 412,37 & $<0,001$ & 24,68 & $<0,001$ \\
\hline $\mathrm{D} \times \mathrm{T}$ & 24,74 & $<0,001$ & 32,69 & $<0,001$ & 4,22 & 0,013 \\
\hline \multicolumn{7}{|l|}{ Corte 4} \\
\hline Tipo (T) & 40,81 & $<0,001$ & & & & \\
\hline Dosis (D) & 116,52 & $<0,001$ & & & & \\
\hline $\mathrm{D} \times \mathrm{T}$ & 7,41 & $<0,001$ & & & & \\
\hline \multicolumn{7}{|l|}{ Total } \\
\hline Tipo (T) & 86,55 & $<0,001$ & 79,06 & $<0,001$ & 69,74 & $<0,001$ \\
\hline Dosis (D) & 652,36 & $<0,001$ & 699,57 & $<0,001$ & 8,72 & 0,002 \\
\hline $\mathrm{D} \times \mathrm{T}$ & 13,20 & $<0,001$ & 14,15 & $<0,001$ & 4,13 & 0,015 \\
\hline
\end{tabular}

${ }^{1} \mathrm{ERNF}=[(\mathrm{N}$ absorbido tratamiento $-\mathrm{N}$ absorbido testigo $) \mathrm{x}$ (dosis de $\mathrm{N}$ aplicado)-1] $\mathrm{x} 100$.

${ }^{1} E N R F=[($ absorbed $N$ treatment - absorbed $N$ control $) \times($ applied $N$ dose $)-1] x 100$. 
la realizamos con la clinoptilolita- $\mathrm{NH}_{4}$. En el segundo corte, son los tratamientos fertilizados con clinoptilolita- $\mathrm{NH}_{4}$ los que registraron el mayor contenido de NT, en relación a los restantes tipos de fertilizantes usados. En este corte, la urea sólo presentó diferencias significativas con la clinoptilolita-urea para la dosis de $120 \mathrm{~kg} \cdot \mathrm{ha}^{-1}$. En el tercer corte de $L$. multiflorum, la clinoptilolita- $\mathrm{NH}_{4}$ fue el tipo de fertilizante que permitió aumentar el NT para todas las dosis empleadas. En el mismo corte de L. multiflorum se observó que la utilización de clinoptilolita-urea permitió aumentar el NT en relación a la urea para la dosis de $180 \mathrm{~kg} \cdot \mathrm{ha}^{-1}$ (Tabla 2). Esto indicaría que la dinámica de desorción de las clinoptilolitas intercambiadas (clinoptilolitas- $\mathrm{NH}_{4}$ ) fue más rápida a la de las formas ocluidas (clinoptilolita-urea). El proceso de liberación de los iones $\mathrm{NH}_{4}^{+}$adsorbidos internamente en la clinoptilolita es un proceso de difusión controlada, que responde al modelo de Freundlich modificado (Kithome et al., 1998).

En ensayos de incubación, se ha demostrado que la dinámica de liberación de sales ocluidas fue más lenta que la liberación de iones intercambiados (Park y Komarnemi, 1998). Asimismo, y en concordancia con los resultados obtenidos en el presente estudio, determinaron que la fracción retenida en las formas ocluidas fue mayor a la de las formas intercambiadas. En el proceso de preparación de la clinoptilolitaurea, el ingreso de una molécula en los canales de la clinoptilolita produce la modificación de los ángulos de la misma, pudiendo inclusive cambiar los tipos de enlace (simples y dobles) y generándose varios sitios de atracción entre la molécula de urea y la clinoptilolita. Dicho procesoexplicaría la disminución de la velocidad de desorción de la urea en relación al $\mathrm{NH}_{4}{ }^{+}$(Park y Komarneni, 1998). Asimismo, no toda la urea ocupa los canales de la clinoptilolita, sino que existe la posibilidad de que una fracción de la urea quede adherida en la superficie externa de dicho mineral, existiendo, por lo tanto, una fracción fácilmente disponible similar a la urea granulada .

\section{Eficiencia de recuperación del nitrógeno}

Según los resultados obtenidos, existió una marcada diferencia en la eficiencia de recuperación entre urea y las clinoptilolitas. Se registró una interacción significativa $(\mathrm{p}<0,013)$ entre el tipo de fertilizante y la dosis de nitrógeno aplicada en el primer y tercer corte, como asimismo la interacción fue también significativa para ERNF (Cuadro 3). Según los resultados obtenidos la clinoptilolita- $\mathrm{NH}_{4}$ aumentó la ERNF en dosis de 120 y $180 \mathrm{~kg} \cdot \mathrm{ha}^{-1}$ de nitrógeno, en relación con la fertilización con urea. Con $60 \mathrm{~kg} \cdot \mathrm{ha}^{-1}$ de nitrógeno la ERNF fue similar (Figura 2). Esto indicaría que el aumento de la eficiencia de uso del fertilizante nitrogenado al utilizar clinoptilolita intercambiada con $\mathrm{NH}_{4}^{+}$se podría atribuir a la relación suelo:clinoptilolita. En el presente ensayo se trabajó con una sola clinoptilolita intercambiada; por lo tanto, el aumento de la dosis de nitrógeno implica un aumento de la cantidad de clinoptilolita por maceta. Para las dosis de 60, 120 y $180 \mathrm{~kg} \cdot \mathrm{ha}^{-1}$ de nitrógeno las relaciones $(\mathrm{p} / \mathrm{p})$ suelo:clinoptilolita fueron de 1:0,0003; 1:0,0006 y 1:0,0012. Al analizar trabajos previos, se observó que las relaciones utilizadas en suelos de textura y CIC similar, si bien son muy diversas, todas ellas son mayores a las del presente trabajo. Weber et al (1983) utilizaron una relación de 1:0,056; MacKown y Tucker (1985) trabajaron con relaciones de 1:0,0125 y Pirela et al (1984) lo hicieron con relaciones de 1:0,0033, encontrando todos ellos mejor resultado con clinoptilolita- $\mathrm{NH}_{4}$ que con urea.

La eficiencia de recuperación de nitrógeno de los tratamientos fertilizados con clinoptilolita-urea fue muy baja en relación a los otros tratamientos. Dado que en el ensayo se mantuvo la humedad del suelo a $90 \%$ de la capacidad de campo, y por lo tanto, no hubo lixiviación ni probabilidades de volatilización cuantitativamente importante, se entiende que esta baja recuperación no responde a un aumento de las pérdidas de nitrógeno, sino que estaría dada por la capacidad de retención de las moléculas de urea en los canales de la clinoptilolita. Como se explicó anteriormente, el proceso de fusión de la urea para la preparación de clinoptilolitaurea posibilita la ubicación de dicho compuesto en sitios internos de los canales, donde queda retenida por atracción electrostática, y en sitios externos donde queda adherida a la superficie 


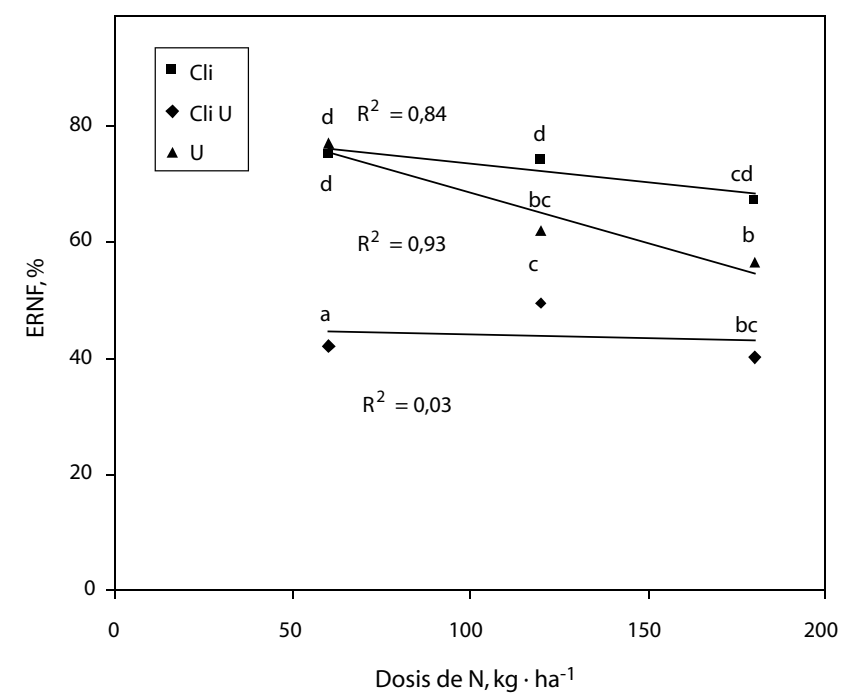

Figura 2. Eficiencia de recuperación del fertilizante (ERNF) aplicado a Lolium multiflorum en todos los tratamientos. Promedios en cada dosis de nitrógeno $(\mathrm{N})$ seguidos por iguales letras no son estadísticamente diferentes entre sí según la prueba de comparación múltiple de medias LCD $(\mathrm{p}<0,05)$. Cli = clinoptilolita-NH4, Cli U = clinoptilolita-urea y U = urea. Figure 2. Efficiency of the retrieval of nitrogen (ENRF) from nitrogen fertilizer applied to Lolium multiflorum cv. Grandeza. Means for each nitrogen $(N)$ doses followed by the same letters are not statistically different from each other according to the least significant differences $(L S D)$ test $(p=0.05)$. Cli $=$ Clinoptilolite-NH4, Cli $U=$ Clinoptilolite-urea, and $U=$ urea .

del mineral. El nitrógeno disponible proviene fundamentalmente de la urea adherida en la superficie externa y en los bordes de dicho material. La urea que ingresó en los canales de la clinoptilolita no estaría disponible en el ciclo del cultivo, quedando ocluida en dichos canales (Park y Komarnemi, 1998).

En función de los resultados obtenidos es posible concluir que el uso de clinoptilolita$\mathrm{NH}_{4}$ aumentó la producción de materia seca de $L$. multiflorum en las condiciones de cultivo del ensayo, respecto de igual dosis aplicada de nitrógeno en forma de urea. Asimismo el uso de este tipo de fertilizante permitió alcanzar producciones de materia seca similares a las obtenidas con urea utilizando menores dosis de nitrógeno.

La utilización de clinoptilolita- $\mathrm{NH}_{4}$ permitió aumentar la eficiencia de recuperación del fertilizante cuando se utilizaron dosis mayores a $120 \mathrm{~kg} \cdot \mathrm{ha}^{-1}$ de nitrógeno. En consecuencia, existiría una fracción remanente menor de nitrógeno en el suelo, susceptible a ser lixiviada o volatilizada, lo cual implicaría menor impacto ambiental de la práctica de la fertilización nitrogenada.

La clinoptilolita-urea produjo rendimientos de materia seca inferiores o similares a la utilización de urea a una misma dosis de nitrógeno aplicada. Sin embargo originó una disminución significativa de la eficiencia de recuperación del nitrógeno del fertilizante en las condiciones del ensayo.

\section{Resumen}

Las propiedades relacionadas con el intercambio catiónico y la selectividad iónica que poseen las clinoptilolitas ha motivado el estudio de su aplicación en el ámbito agropecuario. En trabajos previos se demostró una alta potencialidad de estos minerales como vehículo de fertilizantes nitrogenados, con la finalidad de disminuir problemas ambientales y aumentar la eficiencia de los fertilizantes. El objetivo de este trabajo fue evaluar la producción de materia seca y la recuperación de nitrógeno del fertilizante de un cultivo de Lolium multiflorum cv. Grandeza, fertilizado con clinoptilolita- 
$\mathrm{NH}_{4}$ (forma intercambiada), clinoptilolita-urea (forma ocluida) y urea granulada, mantenido en macetas en invernáculo. Se fertilizó en dosis equivalentes a $0,60,120$ y $180 \mathrm{~kg} \cdot \mathrm{ha}^{-1}$ de nitrógeno y se evaluó el rendimiento de materia seca y la recuperación de nitrógeno del fertilizante a través del análisis de los cuatro cortes de L. multiflorum cv. Grandeza. Según los resultados obtenidos, la utilización de la clinoptilolita- $\mathrm{NH}_{4}$ produjo un aumento de rendimiento para todas las dosis de nitrógeno utilizadas. Asimismo, produjo un aumento en la eficiencia de recuperación del nitrógeno del fertilizante al emplear dosis de 120 y $180 \mathrm{~kg} \cdot \mathrm{ha}^{-1}$ de nitrógeno. La utilización de clinoptilolitaurea presentó menores rendimientos, estadísticamente significativos respecto de los tratamientos fertilizados con urea solo en la dosis menor. Asimismo, la eficiencia de recuperación del nitrógeno fue menor a la de los restantes tratamientos.

Palabras clave: Desorción de cationes, eficiencia, oclusión de moléculas.

\section{Literatura citada}

Allen, E.R., L. Hossner, D. Ming y D. Henninger. 1996. Release rates of phosphorus, ammonium, and potassium in clinoptilolite-phosphate rock systems. Soil Science Society of America Journal 60:1467-1472.

Ando, H., C. Mihara, K. Kakuda y G. Wada. 1996. The fate of ammonium nitrogen applied to flooded rice as affected by zeolite addition. Soil Science and Plant Nutrition 42:531-538.

Andriulo, A., C. Ferreira, C. Nasal, M. Rivero, F. Abrego, M. Bueno, F. Rimatori, M. De la Cruz, M. Venancio y R. Giacosa. 2000. Nitratos en el agua subterránea del partido de Pergamino. Efecto a largo plazo de la agricultura continua y a corto plazo de la producción intensiva de granos. Actas del XVII Congreso Argentino de la Ciencia del Suelo, Mar del Plata, Argentina, 11 al 14 de abril.

Babaririck, K.A. y H. Pirela. 1984. Agronomic and horticultural uses of Zeolites: review.. Pages 93-103. In: W.G. Pond y F.A. Mumptom (eds). Zeo-agriculture. Use of Natural Zeolites in Agriculture and Aquiculture. Westview Press, Boulder, CO, USA. 264 pp.

Bolado Rodríguez, S, A. Alonso y J. Alvarez-Benedí. 2003. Caracterización de procesos acoplados de adsorción, transformación y volatilización de $\mathrm{N}$ en suelos fertilizados con urea. Páginas 185192. En: J. Alvarez-Benedí y P. Marinero (eds.). Estudios de la Zona no Saturada del Suelo, v. VI. Valladolid, España.

Chelishehev, N., N. Volodin y V. Kryukov. 1988. Ion Exchange Properties of Natural high Silica Zeolites. Nauka (ed.), Moscu. 174 pp.

Costa, J. L. 1997. Riego y Medio Ambiente. Calidad de aguas para riego. Páginas 7-14. En: Seminario de Riego. Regional Buenos Aires Sur (ed.), Estación Experimental Agropecuaria Balcarce, INTA. Mar del Plata, Argentina.

Ferguson, G. y I. Pepper. 1987. Ammonium retention in sand amended with clinoptilolite. Soil Sciences Society of American Journal 51:231-234.

Griffith, S.M. y Streeter D.J. 1994. Nitrate and ammonium nutrition in ryegrass: changes in growth and chemical composition under hydroponic conditions. Journal of Plant Nutrition 17:71-81.

Inglezakis, V., M. Loizidou y H. Grigoropoulou. 2004. Ion exchange studies on natural and modified zeolites and the concept of exchange site accessibility. Journal of Colloid and Interface Science. 275:570-576.

Kithome, M., J. W. Paul, L. M. Lavkulich, y A. A. Bomke. 1998. Kinetics of Ammonium Adsorption and Desorptionby the Natural Zeolite Clinoptilolite. Soil Sciences Society of American Journal 62:622-629.

Kolyagin, Yu, S. y O. A. Karasev. 1999. Root nutrition and the quality of sugarbeet. Sakharnaya Svekla (Bulgaria) 6:11-12.

Lewis, M. D., F.D. Moore y K.L. Goldberry. 1984. Ammonium-exchanged clinoptilolite and granulated clinoptilolie with urea as nitrogen fertilizers. Pages 123-137. In: W.G. Pond and F.A. Mumptom (eds.). Zeo Agriculture: Use of Natural Zeolites in Agriculture and Aqcuaculture. Westview Press, Boulder, CO, USA. 264 pp.

Loboda, B. 1999. Agroecological assessment of using substrates from zeolite-containing rocks in greenhouse grown sweet pepper. Agrokhimiya (Rusia) 2:67-72.

MacKown, C. y T. Tucker. 1985. Ammonium nitrogen movement in a coarse-textured soil amended with zeolite. Soil Science Society of America Journal 49:235-238.

Malavolta, E., G. Vitti y S. de Oliveira. 1989. Avaliaçáo do estado nutricional das plantas: Principios e aplicaçoes. Associaçáo Brasileira para Pesquisa da Potassa e do Fosfato. Piracicaba, SP, Brasil. 325 pp.

Mendenhall, W., R. Scheaffer y D. Wackerly 1986. Estadística Matemática Con Aplicaciones. 
Grupo Editorial Iberoamericana. California, EUA. 251 pp.

Park, M. y S. Komarneni. 1998. Ammonium nitrate occlusion vs. nitrate ion exchange in natural zeolites. Soil Science Society of America Journal 62:1455-1459.

Pirela, H.G., D.G. Westfall y K.A. Babarick. 1984. Use of clinoptilolite in combination with nitrogen fertilization to increase plant growth. Pages 65-76. In: W.G. Pond and F.A. Mumptom (eds.). Zeo Agriculture: Use of Natural Zeolites in Agriculture and Aqcuaculture. Westview Press, Boulder, CO. USA. 264 pp
Postnikov, A.V., G. A. Romanov, B. Loboda y A.V. Zekunov. 1996. A good substrate for green crops. Kartofel' i Ovoshchi 5:21-22.

Soca M., Castellanos J. y Febles J. 2004. Efecto de la zeolita en la eficiencia de los fertilizantes químicos, fertilizantes y enmiendas de origen mineral. Panorama Minero (Buenos Aires, Argentina) 14:261-268.

Watson, C. J. 1986. Preferential uptake of ammonium nitrogen from soil by ryegrass under simulated spring conditions. Journal of Agricultural Science 107:171-177. 